\title{
Eye Blink Artefact Removal of Single Frontal EEG Channel Algorithm using Ensemble Empirical Mode Decomposition and Outlier Detection
}

\author{
M.N.A.H. Sha'abani ${ }^{a, d, *}$, N. Fuad ${ }^{b, c}$, N. Jamal ${ }^{b}$ \\ a Centre for Diploma Studies, Universiti Tun Hussein Onn Malaysia, Malaysia; \\ ${ }^{b}$ Faculty of Electrical and Electronic Engineering, Universiti Tun Hussein Onn \\ Malaysia, Malaysia; ' Computational, Signal, Imaging and Intelligent Focus Group \\ (CSII), Universiti Tun Hussein Onn Malaysia, Malaysia; ${ }^{d}$ Microcontroller Technology \\ for loT Focus Group (MTIT), Universiti Tun Hussein Onn Malaysia, Malaysia
}

Abstract Recently, the emergence of various applications to use EEG has evolved the EEG device to become wearable with fewer electrodes. Unfortunately, the process of removing artefact becomes challenging since the conventional method requires an additional artefact reference channel or multichannel recording to be working. By focusing on frontal EEG channel recording, this paper proposed an alternative single-channel eye blink artefact removal method based on the ensemble empirical mode decomposition and outlier detection technique. The method removes the segment of the potential eyeblinks artefact on the residual of a pre-determined level of decomposition. An outlier detection technique is introduced to identify the peak of the eyeblink based on the extreme value of the residual signal. The results showed that the corrected EEG signal achieved high correlation, low RMSE and have small differences in PSD when compared to the reference clean EEG. Comparing with an adaptive Wiener filter technique, the corrected EEG signal by the proposed method had better signal-to-artefact ratio.

Keywords: electroencephalogram, eye blink removal, single-channel, EEMD

*For correspondence: nhafiz@uthm.edu.my

Received: 23 June 2021 Accepted: 11 Nov 2021

(C) Copyright Sha'abani et al. This article is distributed under the terms of the Creative Commons Attribution License, which permits unrestricted use and redistribution provided that the original author and source are credited.

\section{Introduction}

Electroencephalogram (EEG) is one of the techniques to record brain signals by using electrodes affixed on the scalp following the standard 10-20 systems [1]. EEG is non-invasive and safe, making it the most preferable modality for recording brain activities in various clinical and non-clinical applications [2]. For example, epilepsy diagnosis [3, 4], brain-computer interface [5, 6] and cognitive studies [7, 8]. Earlier EEG experiments required plenty of preparation with many electrodes and wires attached to the scalp. In recent years, the emergence of other applications on using EEG evolves the EEG device into portable with fewer electrodes, ergonomic and wireless. This innovation allows the EEG experiments to be performed outside of the laboratory environment [9]. However, the drawback of fewer electrodes is the processing of artefact removal becomes challenging because of fewer signal sources. Thus, this study will address this issue by proposing an alternative method to remove artefact, focusing on a single frontal EEG channel.

EEG recorded on frontal lobe area usually contaminated by eye blink artefact [10]. Eye blinks can produce a very large potential compared to the EEG signal, lasting between 50 and 500 milliseconds at a rate of 20 times per minute [11]. Due to its high magnitude, it is crucial to remove the eye blink signal 
as it will lead to undesirable results in the EEG signal analysis [12]. The easiest way to prevent eye blink artefacts is by asking the participant to avoid blinking. Though, it is not practical for a subject to limit eye blinking for a long period of an experiment [13]. Therefore, the eye blink signal will always be present in the EEG recording. The simplest method is by removing the contaminated EEG segment or using a bandpass filter to remove the $0-12 \mathrm{~Hz}$ electrooculography (EOG) frequency band. However, since the eye blink overlaps with the EEG signal, these methods may lead to the loss of some important EEG data [14].

The traditional method to suppress eye blink artefact is by using regression and adaptive filter technique. Both methods require an artefact reference channel, therefore, it is not suitable to be used for mobile EEG devices since most of them do not provide the reference channel. The other way is by decomposing the EEG signal into several components without the aid of artefact reference channel. The most popular decomposition technique is the independent component analysis (ICA) [15]. ICA assumes that the recorded EEG signal is an instantaneous linear mixture of several independent sources (i.e., pure EEG signal and artefact signals). Thus, ICA decomposed the EEG signal into several independent components (ICs) and then discarded the ICs containing the potential artefact. The remaining ICs are reconstructed as the corrected EEG [16]. However, since ICA requires a sufficient number of EEG channels, it is not working well when using a single or few numbers of EEG channels [17].

Otherwise, wavelet transform (WT) and empirical mode decomposition (EMD) offers a good technique in decomposing non-stationary EEG signals even using a single EEG channel [16]. WT transforms the EEG signal from the time domain to a time-frequency domain into a set of coefficients based on a scaled and shifted process of a mother wavelet function [18]. Briefly, the EEG signal is filtered into a low pass subband (i.e., approximation) and a high pass subband (i.e., detail). After decomposition, components that contain artefacts are discarded and the corrected EEG signal is obtained by adding up the detail coefficients at each level of decomposition. Though, WT is unable to remove artefacts that overlap with EEG signals in spectral properties [18, 19]. Moreover, without having prior knowledge of the interest signal, it would be difficult to choose WT's optimal basis function [20].

Huang et.al. [21] has proposed a heuristic technique to decompose non-stationary and non-linear signals called EMD. EMD decomposes a signal into a series of intrinsic mode functions (IMF) through an iterative method called the sifting process. In that process, the local extremes of a signal are identified and interpolated to form an upper and lower envelope. Then, the mean envelope is subtracted and assigned as the IMF. The sifting process is stopped when the residual signal exhibit a monotonic function [21]. IMF can be defined according to the following conditions: (i) the total number of local extrema and the number of zero-crossings must be equal or differ at most by one, and (ii) the mean value of upper and lower envelope is zero. After decomposition, IMFs containing artefact signals can be discarded. The corrected signal is obtained by summing up the remaining IMFs. Unlike WT, EMD is a data-driven algorithm, it decomposes nonlinear and non-stationary signals naturally without a priori knowledge of the signal $[22,23]$.

According to the above discussion, EMD-based method offers a good decomposition on removing eye blink artefacts based on the following conditions: (i) feasible for a single or few numbers of EEG channel, (ii) do not require artefact reference channels (i.e., prior knowledge) and (iii) completely a data-driven method. The original EMD method is sensitive to noise and faced a mode mixing problem. Wu and Huang [24] have overcome this issue by proposing a noise-assisted method called the ensemble empirical mode decomposition (EEMD), which gives a major improvement of the original EMD. Hence, this study is proposed to implement EEMD to decomposed EEG signal and removing the eye blink artefact with the aid of outlier detection technique. The detailed procedures of the proposed method are described in the methodology section. 


\section{Data description Modelling of eye blink artefact}

The shape of an eye blink artefact was generated by averaging 10 samples of eye blink spikes from a raw EEG signal. The raw EEG signal was recorded on the frontal lobe (i.e., AF3) at $128 \mathrm{~Hz}$ of sampling rate [25]. Initially, the raw EEG signal was detrended to remove the DC offset. Then, a high pass filter with $0.5 \mathrm{~Hz}$ of cut-off frequency was used to remove the low-frequency noises. Later, the eye blink artefacts were identified and extracted manually by referring to the landmark properties of an eye blink as described in [26]. Figure 1 depicts the segmentation and the average of the eye blink artefact. The average eye blink artefact was then composed with a series of 10 seconds of white noise signals such

$$
c(t)=s^{*} \text { average eye blink }+n(t)
$$

where $c(t)$ is the created eye blink artefact signal, $s$ is a random value to scale the amplitude of the eye blink artefact and $n(t)$ is the series of the white noise signal. For analysis purposes, the signal-to-noise ratio (SNR) of the white noise signal is varied in the experimental.

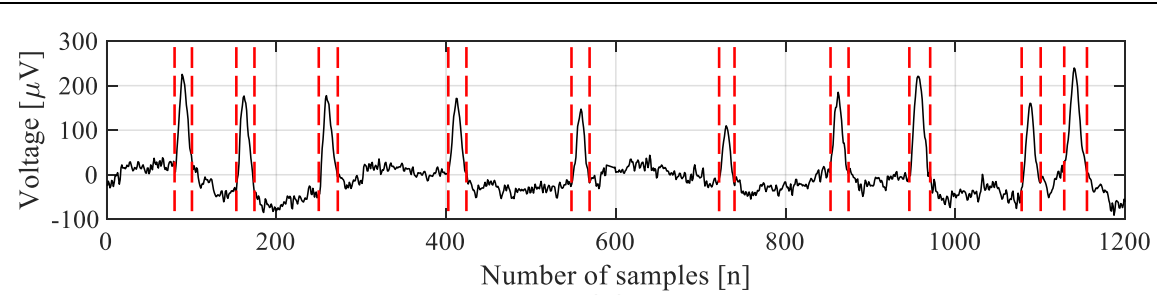

(a)

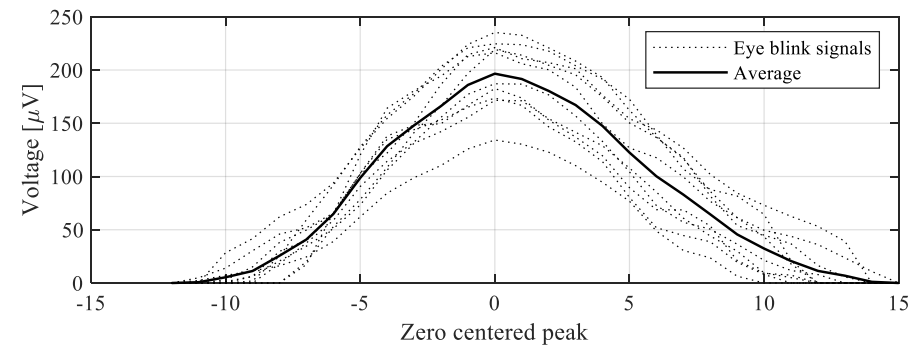

(b)

Figure 1. (a) Segmentation of eye blink artefacts, (b) the average of eye blink artefact.

\section{Modelling of contaminated EEG signal}

The modelling of the contaminated EEG signal was composed of a clean EEG signal and the created eye blink artefact signal. The clean EEG signal was taken from [27], particularly at AF3 channel, within the resting state segment with closed eyes. It is claimed to be an artefact-free EEG, thus it will be used as the reference of a clean EEG signal for this study. Since the reference EEG signal was recorded at $500 \mathrm{~Hz}$, the signal was downsampled to $128 \mathrm{~Hz}$ to match the sampling frequency of $c(t)$. The model of the contaminated EEG signal was defined as follows:

$$
y(t)=x(t)+c(t)
$$

where $y(t)$ is the model of the contaminated EEG signal, and $x(t)$ is the reference clean EEG. Figure 2 illustrates the steps to model the contaminated EEG signal. Note that the average eye blink artefact was duplicated ten times with different amplitude and time scales. 
(a) Clean EEG signal, $x(t)$

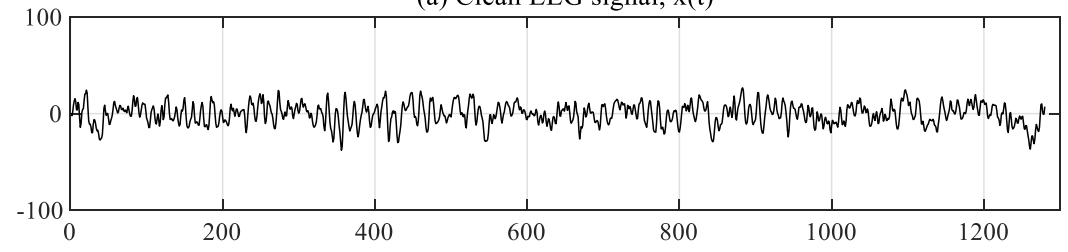

(b) Eye blink signal, c(t)

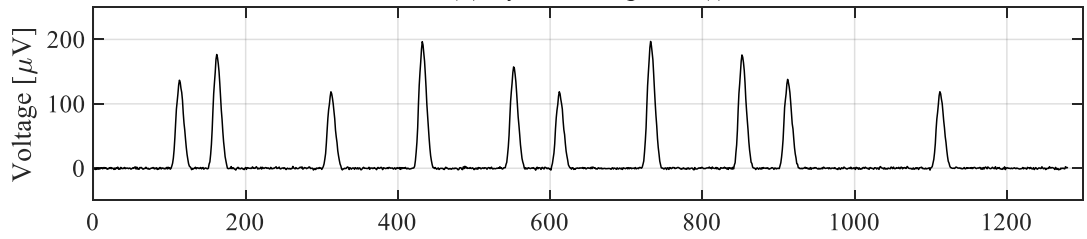

(c) Contaminated EEG signal, $y(t)$

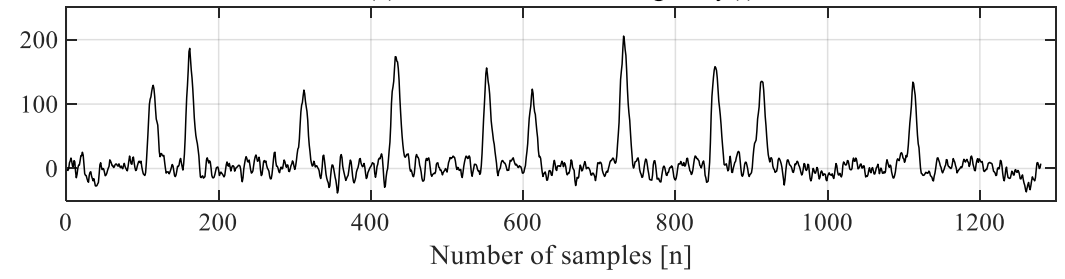

Figure 2. Steps to model the contaminated EEG signal.

\section{Methodology}

\section{The proposed method}

Figure 3 shows the framework of the proposed method. Initially, a normalized EEG signal is decomposed using EEMD with a pre-determined level of IMF. From its residual signal, the extreme values are identified. Note that, the residual term in this work refers to the summation of remaining unselected IMFs and the decomposition residual. An outlier detection technique is established to identify extreme values that differ significantly from the typical amplitude of an EEG signal. The identified outlier is assumed as the peak of an eye blink. Next, the segment of the eye blink artefacts is removed from the residual signal. The removed segment data is then reconstructed by using the cubic spline interpolation method. Finally, the decomposed EEG signal is reconstructed by summing up all the IMFs and the artefact-free residual signal to obtain the corrected EEG signal.

The detailed algorithm of the proposed method is briefly explained as follows:

1. Signal normalization: The EEG signal is normalized using the standard deviation method:

$$
z(t)=\frac{y(t)}{\sigma}
$$

where $z(t)$ is the normalized EEG signal and $\sigma$ is the standard deviation of signal $y(t)$. 


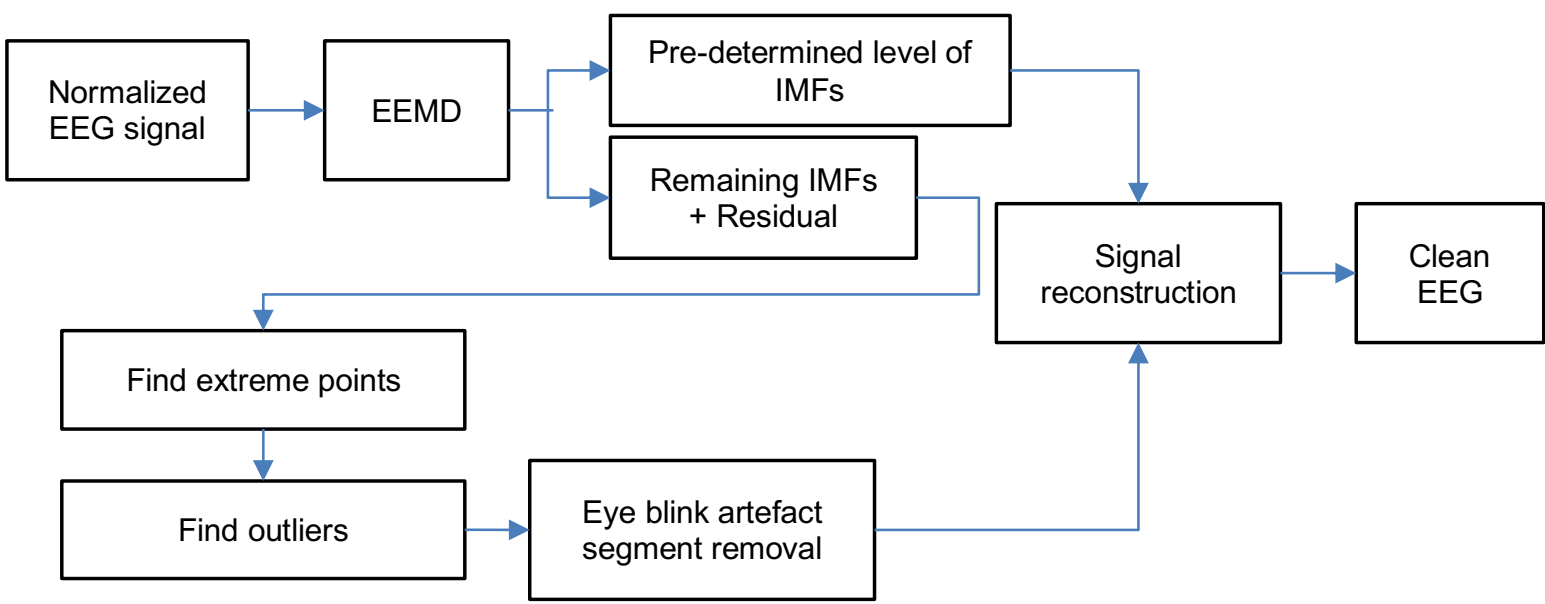

Figure 3. The framework of the proposed eye blink artefact removal technique.

2. Ensemble empirical mode decomposition: The decomposition processes of the contaminated EEG signal, $y(t)$ using EEMD are as follows:

i. Add a series of white noise, $\omega_{n}(t)$ with an amplitude, a to $z(t)$, such $z_{n}(t)=z(t)+a^{*} \omega_{n}(t)$. In this study, the amplitude of the added noise, a was set to 0.4 .

ii. Find the local extrema of $z_{n}(t)$ such that local minima, $e_{\min }(t)$ and local maxima, $e_{\max }(t)$.

iii. Construct an upper envelope, $u(t)$ and lower envelope, $b(t)$ by connecting the series of $e_{\min }(t)$ and series of $e_{\max }(t)$ using the cubic spline interpolation method.

iv. Calculate the mean of the envelopes, $m(t)=\frac{u(t)+b(t)}{2}$.

v. Compute the difference, $d(t)=z_{n}(t)-m(t)$.

vi. Repeat steps (ii) to (v) until $d(t)$ satisfies the basic conditions of IMF. If $d(t)$ meets the conditions of IMF, assign $d(t)$ as the first IMF such $I M F_{1}=d(t)$. This repetition is called the sifting process.

vii. Generate the residual such that $r(t)=z_{n}(t)-I M F_{1}$.

viii. Repeat steps (ii) to (viii) to find the next IMFs by replacing $z_{n}(t)$ as $r(t)$. Note that, in this study, the process is stopped at a pre-determined level of IMF.

ix. Repeat steps (i) until (viii) for $n$ number of ensembles with different white noise series, $\omega_{n}(t)$, and obtain the ensemble mean of the corresponding IMFs as the result. In this study, the number of ensembles used was 200.

Determination of decomposition level

Referring to step (viii), the level of extracted IMF was determined by finding the highest correlation between the residual, $r(t)$ and the created eye blink artefact signal, $c(t)$. An analysis was conducted by repeating the decomposition process with different level of extracted IMF. Then, the correlation was computed for each decomposition process as shown in Table 1. The highest correlation was 0.973, obtained from the residual signal when the level of extracted IMF was 3. Hence, for future EEMD decomposition, this value is preferred.

Table 1. Correlation of corresponding IMFs and the created eye blink artefact signal, $c(t)$.

\begin{tabular}{cc}
\hline Number of IMF extracted from signal, $y(t)$ & Correlation at residual signal, $r(t)$ \\
\hline 1 & 0.959 \\
2 & 0.964 \\
3 & 0.973 \\
4 & 0.929 \\
5 & 0.821 \\
\hline
\end{tabular}

Figure 4 shows the EEMD decomposition of the signal $y(t)$ into three level of IMFs. The $r$ value is the 
Pearson's correlation of IMFs and residual with the created eye blink signal, $\mathrm{c}(\mathrm{t})$. The correlation $r$ indicates that the residual, $r(t)$ is approximately similar to $c(t)$. Hence, it is feasible to remove eye blink artefact from $r(t)$ of three extracted IMFs. The peak of the eye blink is identified using the proposed outlier detection method as explained in the next step.

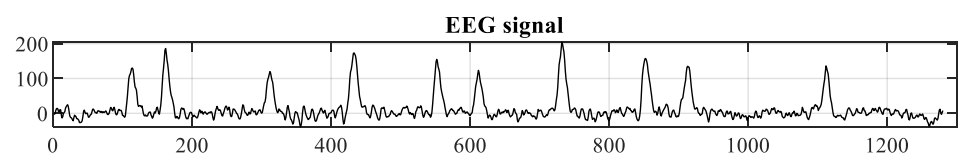
IMF1

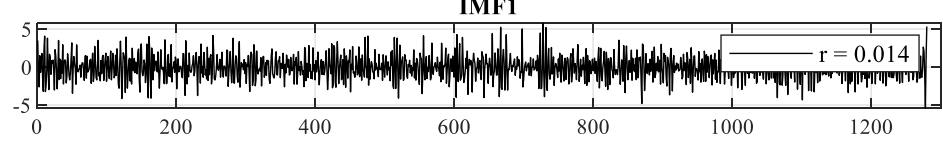
IMF2

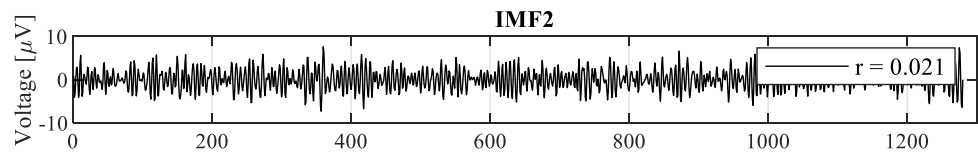
IMF3
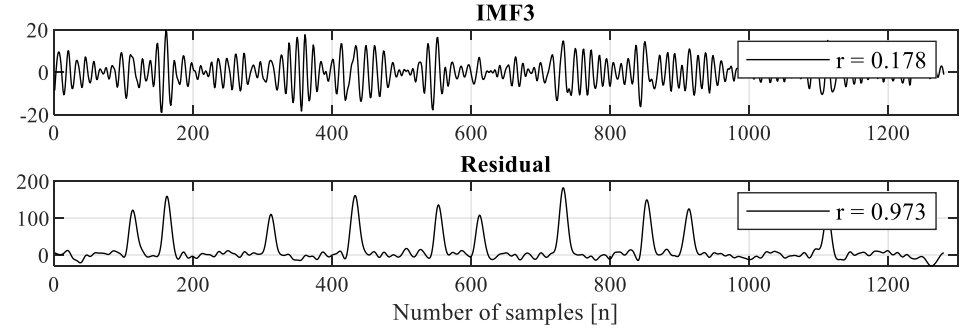

Figure 4. EEMD decomposition of the contaminated EEG signal, $y(t)$ with three level of IMFs.

3. Outlier Detection: The typical peak-to-peak amplitude range of an EEG signal can reach up to $100 \mu \mathrm{V}$ when measured from the scalp $[10,28]$. Based on this fact, the outlier detection is established to identify an extreme value that has an amplitude difference larger than $100 \mu \mathrm{V}$ from its closer extreme value. The procedures of the detection are explained below:

i. Find extreme values of the residual signal, $r(t)$.

ii. Find the outlier(s) from the extreme values based on the following condition:

Let $E$ be the set of the extreme values with $k$ number of elements.

If $\left(E_{k+1}-E_{k}\right)>100 \mu \mathrm{V}$, assign $E_{k+1}$ as an outlier.

4. Eyeblink artefact segment elimination: The outlier obtained in the previous step is assumed as the peak of the potential eye blink artefact. Hence, the lower base data points of the detected eye blink spike are taken from the nearest local minima on both sides (i.e., $E_{k}$ and $E_{k+2}$ ) of the detected peak, $E_{k+1}$. Finally, the eye blink signal artefacts are removed from $r(t)$ by eliminating data points from $E_{k}$ to $E_{k+2}$. The residual signal was then reconstructed using a piecewise cubic spline interpolation method. Figure 5 illustrates the steps on removing the potential eye blink spikes.

5. Signal reconstruction: This study assumed $y(t)$ was only contaminated with eye blink artefacts. Thus, the corrected EEG can be obtained by adding the IMFs and the reconstructed residual signal such

$$
Y(t)=\sum_{i=1}^{j} I M F_{i}+r_{j}
$$

where $Y(t)$ is the corrected EEG signal, IMFi is the set ensemble means of IMF and $r_{j}$ is the reconstructed residual signal $r(t)$. The corrected EEG signal was then evaluated using performance metrics described in the next section. 

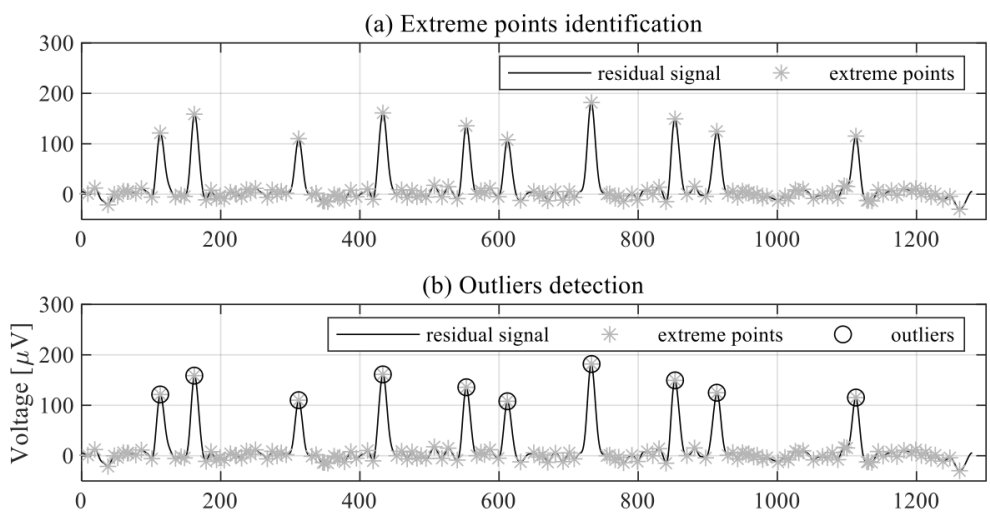

(c) Eye blink signal removal

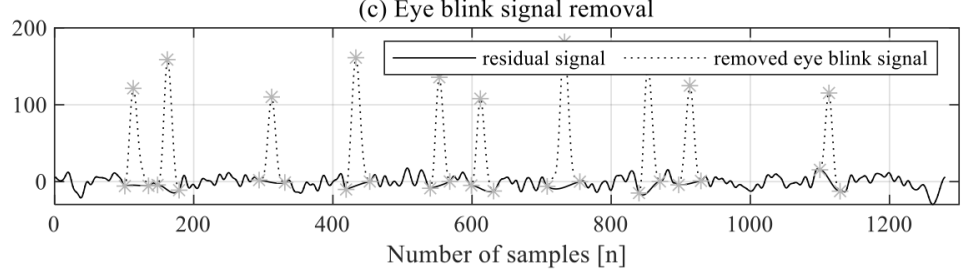

Figure 5. The processes involved in removing eye blink spikes.

\section{Performance metrics}

The performance of the proposed method was evaluated by measuring the similarity between the corrected EEG, $Y(t)$ and the reference clean EEG signal, $x(t)$ using Pearson's correlation, root mean square error (RMSE) and power spectral density (PSD) differences. Pearson's correlation quantifies the relationship between the corrected and the original clean EEG signal. While RMSE calculates the differences (i.e., error) between $Y(t)$ and $x(t)$. The Pearson's correlation and the RMSE are defined as follow:

$$
\begin{gathered}
r=\frac{\sum_{t=1}^{N}[x(t)-\bar{x}][Y(t)-\bar{Y}]}{\sqrt{\sum_{t=1}^{N}[x(t)-\bar{x}]^{2} \sum_{t=1}^{N}[Y(t)-\bar{Y}]^{2}}} \\
R M S E=\sqrt{\frac{\sum_{t=1}^{N}[x(t)-Y(t)]^{2}}{N}}
\end{gathered}
$$

where $N$ is the signal length, and $\bar{x}$ and $\bar{Y}$ are the mean of signal $x(t)$ and $Y(t)$ respectively.

The power spectral density (PSD) differences, $e_{p s d}$ between $Y(t)$ and $x(t)$ across the brainwave frequencies (i.e., $\delta(0.5-4 \mathrm{~Hz}), \theta(4-8 \mathrm{~Hz}), \alpha(8-13 \mathrm{~Hz}), \beta(13-30 \mathrm{~Hz})$ and $\gamma(30-45 \mathrm{~Hz}))$ are also determined. The PSD is calculated using Welch's method [29]. A lesser $e_{p s d}$ indicates that the frequency properties of $Y(t)$ are close to $x(t)$.

In addition, the performance of the proposed method has been compared with an adaptive Wiener filter. The purpose of comparing with Wiener filter is to evaluate the feasibility of the proposed method in removing eye blink artefact even not using an artefact reference signal. A signal-to-artefact ratio (SAR) [30] was used to evaluate the performance of both artefact removal methods. SAR is calculated before and after the artefact removal process by using these equations:

$$
S A R_{\text {contaminated }}=10 \log _{10}\left(\frac{s t d[x(t)]}{s t d[x(t)-y(t)]}\right)
$$




$$
S A R_{\text {corrected }}=10 \log _{10}\left(\frac{s t d[x(t)]}{s t d[x(t)-Y(t)]}\right)
$$

where std refers to standard deviation. Method that has higher SAR value indicates a better artefact removal process [31].

\section{Results and discussion}

Figure 6 shows the comparison of the EEG signal before and after the artefact removal process by the proposed method. As can be seen, the PSD of $x(t)$ was altered from P1 to P2 after the eye blink artefact was added into the signal. In P2, most of the power between $0 \mathrm{~Hz}$ and $10 \mathrm{~Hz}$ was coming from the eye blink artefact. In Figure 6 (c), the eye blink artefacts were successfully eliminated from $y(t)$ after the proposed eye blink removal process was implemented. Subsequently, the PSD of the EEG signal was successfully recovered as illustrated in P3.
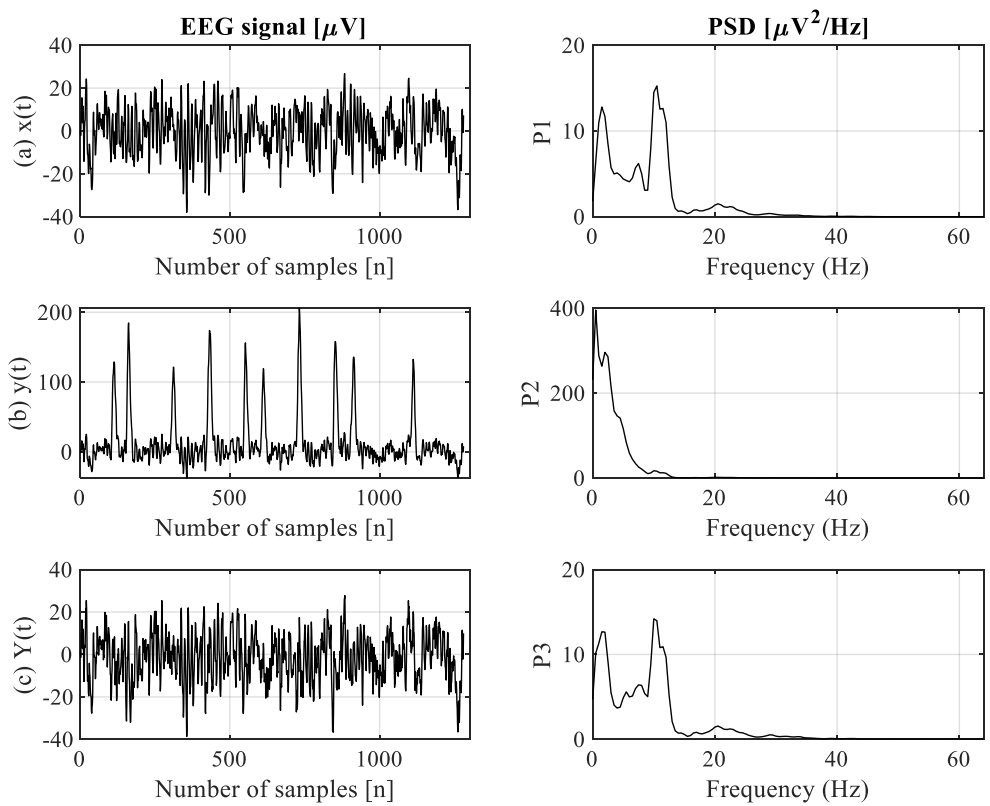

Figure 6. Comparison of time series EEG signal and the PSD between (a) $x(t),(b) y(t)$ and (c) $Y(t)$.

An analysis has been conducted to observe the performance of the proposed method under different SNR conditions. For comparison, an adaptive Wiener filter has been implemented on the same contaminated EEG signal, $y(t)$. Table 2 shows the results of the correlation, $r$ and the RMSE of the analysis. In general, the proposed method showed a higher correlation and less RMSE than the Wiener filter approach. These results indicate that the EEG signal corrected by the proposed method has a strong positive correlation and less distortion than the Wiener filter approach. However, the performance decreased with SNR $=-20 \mathrm{~dB}$.

Table 2. Comparison of correlation and RMSE of the proposed method with the Wiener filter approach.

\begin{tabular}{ccccc}
\hline \multirow{2}{*}{ SNR $(\mathrm{dB})$} & \multicolumn{2}{c}{ Proposed method } & \multicolumn{2}{c}{ Wiener filter } \\
\cline { 2 - 5 } & $r$ & RMSE & 0.54 & RMSE \\
-5 & 0.87 & 5.74 & 0.52 & 9.27 \\
-10 & 0.87 & 6.03 & 0.51 & 9.36 \\
-15 & 0.85 & 6.52 & 0.50 & 9.45 \\
-20 & 0.79 & 7.95 & 0.43 & 9.55 \\
\hline
\end{tabular}


Table 3 shows the comparison of the $e_{p s d}$ by the proposed method and the Wiener filter across the brainwave subbands. In general, the proposed method has a smaller $e_{p s d}$ than the Wiener filter approach. A positive $e_{p s d}$ was observed on the proposed method because there was still a small fraction of the eye blink that exists in the EEG signal after the removal process. However, the amount of the residual eye blink artefact is much smaller than the amount being removed. Meanwhile, the $e_{p s d}$ of the Wiener filter approach showed a negative value, which indicates that the approach excessively removed the EEG signal. This is shown in Figure 7 where the EEG signal corrected by the Wiener filter was degraded, while the proposed method successfully preserved most of the frequency component of the EEG signal.

Table 3. Comparison of $e_{p s d}$ of the proposed method and the Wiener filter at each brainwave subbands.

\begin{tabular}{|c|c|c|c|c|c|c|c|c|c|c|}
\hline \multirow{2}{*}{ SNR } & \multicolumn{5}{|c|}{ Proposed method } & \multicolumn{5}{|c|}{ Wiener filter } \\
\hline & $e_{p s d \_} \delta$ & $e_{p s d}=\theta$ & $e_{p s d \_\alpha}$ & $e_{p s d} \beta$ & $e_{p s d} \gamma$ & $e_{p s d \_} \delta$ & $e_{p s d}=\theta$ & $e_{p s d \_} \alpha$ & $e_{p s d \_} \beta$ & $e_{p s d} Y_{1}$ \\
\hline 0 & +3.55 & +1.82 & +0.64 & +1.18 & +0.93 & -26.25 & $-14 . \overline{71}$ & -23.61 & -9.12 & -0.09 \\
\hline-5 & +5.96 & +1.51 & +1.25 & +2.37 & +1.39 & -26.15 & -14.53 & -24.24 & -9.43 & -0.41 \\
\hline-10 & +7.12 & +1.50 & +1.03 & +2.94 & +3.37 & -26.34 & -14.32 & -24.29 & -9.70 & -0.81 \\
\hline-15 & +7.41 & +0.69 & +3.36 & +11.19 & +9.03 & -25.64 & -14.44 & -24.79 & -9.89 & -0.90 \\
\hline-20 & +11.53 & +2.88 & +3.67 & +32.78 & +24.30 & -26.64 & -14.98 & -29.88 & -9.72 & -0.39 \\
\hline
\end{tabular}
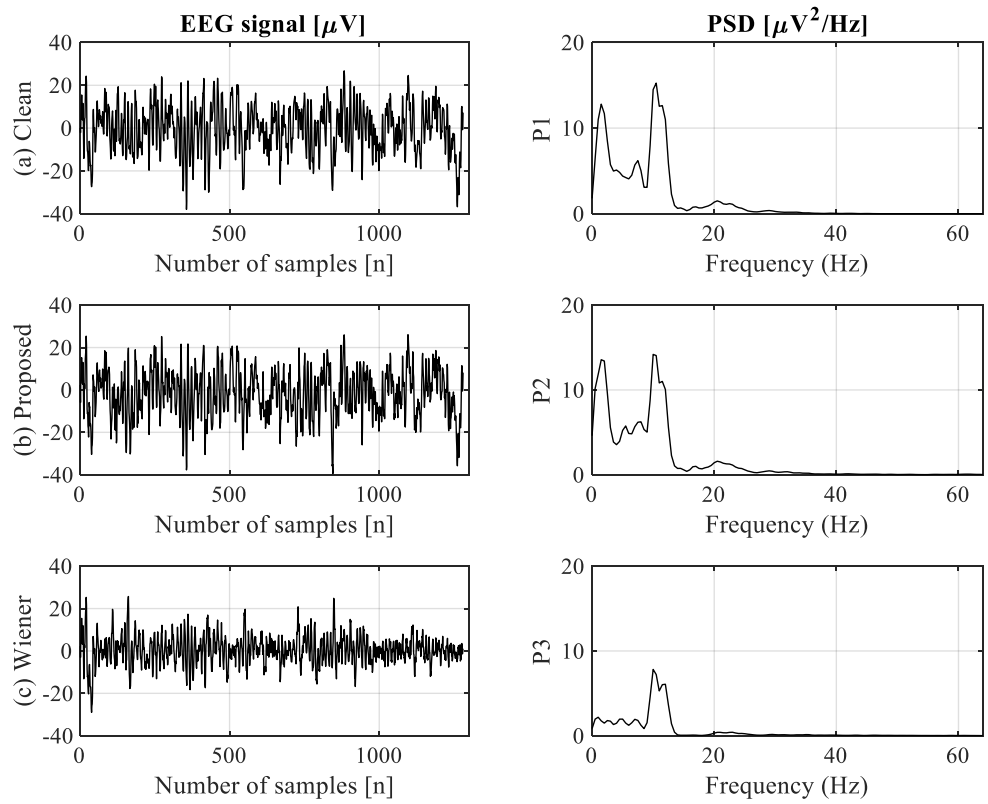

Figure 7. The comparison between (a) the clean EEG signal and the corrected EEG signal by (b) the proposed method and by (c) the Wiener filter at SNR $=0 \mathrm{~dB}$.

Figure 8 shows the SAR of the EEG signal before and after the artefact removal process for the proposed method and the Wiener filter over $0 \mathrm{~dB}$ to $-20 \mathrm{~dB}$ of SNR conditions. Generally, the SAR of the EEG signal was increased after using both eye blink artefact removal processes. Over the SNR conditions, the SAR value of the proposed method decreased as the SNR value decreased, but the Wiener filter approach did not show any significant changes. By comparing SAR values, the proposed method has a better SAR than the Wiener filter approach. This shows that the proposed method outperforms the Wiener filter approach in eliminating the eye blink artefact. However, at SNR $=-20 \mathrm{~dB}$, the SAR of the proposed method was less than the Wiener filter approach. 


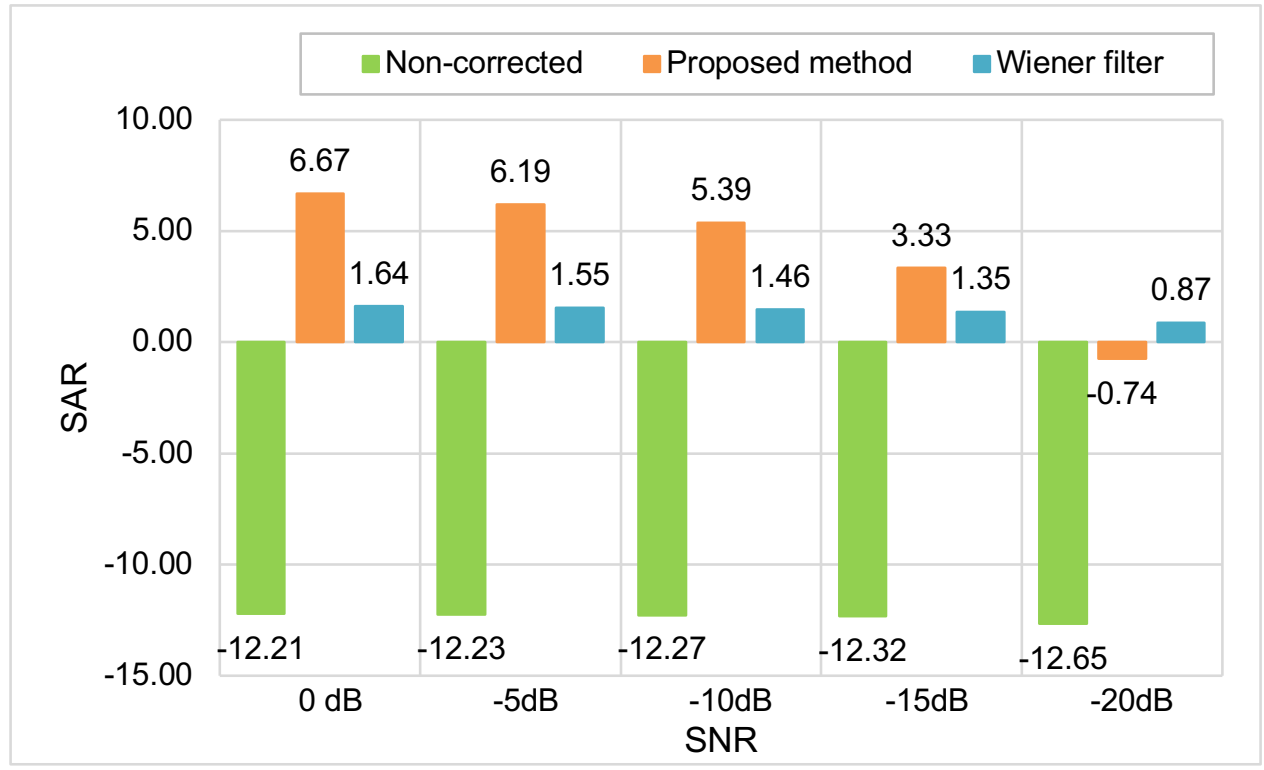

Figure 8. SAR of the EEG signal before and after the eye blink artefact removal over $0 \mathrm{~dB}$ to $-20 \mathrm{~dB}$ of SNR conditions.

\section{Conclusion}

In this paper, the eye blink artefact removal method for EEG signal based on EEMD and outlier detection technique has been proposed. The significance of this method is it can be used on a single EEG channel and does not require an EOG reference channel. The experiment on simulated EEG data demonstrated that the proposed method is capable to remove the eye blink artefact and retain most of the time and frequency component of the EEG data. The proposed method is also compared with the conventional Wiener filter approach. The SAR results showed that the proposed method is better than the Wiener filter approach in removing the eye blink artefact.

\section{Conflicts of interest}

The author(s) declare(s) that there is no conflict of interest regarding the publication of this paper.

\section{Acknowledgments}

The author would like to thank the Universiti Tun Hussein Onn Malaysia for supporting the author a $\mathrm{PhD}$ scholarship. Appreciation also goes to the Centre for Diploma Studies and Faculty of Electrical and Electronic Engineering.

\section{References}

[1] R. W. Homan, J. Herman, and P. Purdy, "Cerebral location of international 10-20 system electrode placement," Electroencephalography Clinical Neurophysiology, vol. 66, no. 4, pp. 376-382, 1987.

[2] M. Rashid, N. Sulaiman, A. P. P. Abdul Majeed et al., "Current status, challenges, and possible solutions of eeg-based brain-computer interface: A comprehensive review," Frontiers in Neurorobotics, Review vol. 14, no. 25,2020

[3] R. Abreu, M. Leite, A. Leal, and P. Figueiredo, "Objective selection of epilepsy-related independent components from eeg data," Journal of Neuroscience Methods, vol. 258, pp. 67-78, 2016.

[4] M. S. Fathillah, R. Jaafar, K. Chellappan et al., "Multiresolution analysis on nonlinear complexity measurement of eeg signal for epileptic discharge monitoring," Malaysian Journal of Fundamental Applied Sciences, vol. 14 , no. 2, pp. 219-225, 2018.

[5] H. Lim and J. Ku, "Multiple-command single-frequency ssvep-based bci system using flickering action video," Journal of Neuroscience Methods, vol. 314, pp. 21-27, 2019. 
[6] M. F. M. Rafi, A. R. A. Harris, T. T. Swee et al., "Brain-computer interface algorithm based on wavelet-phase stability analysis in motor imagery experiment," Malaysian Journal of Fundamental Applied Sciences, vol. 16, no. 2, pp. 236-242, 2020.

[7] N. Fuad, J. Bakar, M. N. Danial, E. Nasir, and M. Marwan, "A comparative study of learning methodology between cognitive and psychomotor for non-dyslexia person via electroencephalogram (eeg)," International Journal of Scientific and Technology Research, vol. 8, no. 7, 2019.

[8] M. Gasah, A. Baharum, and N. H. M. Zain, "Measure learning effectiveness among children using eeg device and mobile application," Indonesian Journal of Electrical Engineering and Computer Science, Review vol. 17, no. 1, pp. 191-196, 2019.

[9] A. J. Casson, "Wearable eeg and beyond," Biomedical Engineering Letters, vol. 9, no. 1, pp. 53-71, 2019.

[10] M. Teplan, "Fundamentals of eeg measurement," Measurement Science Review, vol. 2, no. 2, pp. 1-11, 2002.

[11] S. Halder, M. Bensch, J. Mellinger et al., "Online artifact removal for brain-computer interfaces using support vector machines and blind source separation," Computational Intelligence Neuroscience, vol. 2007, 2007.

[12] S. Sreeja, R. R. Sahay, D. Samanta, and P. Mitra, "Removal of eye blink artifacts from eeg signals using sparsity," IEEE Journal of Biomedical Health Informatics, vol. 22, no. 5, pp. 1362-1372, 2017.

[13] M. K. Islam, A. Rastegarnia, and Z. Yang, "Methods for artifact detection and removal from scalp eeg: A review," Neurophysiologie Clinique/Clinical Neurophysiology, vol. 46, no. 4, pp. 287-305, 2016.

[14] S. Zhang, J. Mclntosh, S. M. Shadli et al., "Removing eye blink artefacts from eeg-a single-channel physiology-based method," Journal of Neuroscience Methods, vol. 291, pp. 213-220, 2017.

[15] P. Comon, "Independent component analysis, a new concept?," Signal Processing, vol. 36, no. 3, pp. 287314, 1994.

[16] X. Jiang, G.-B. Bian, and Z. Tian, "Removal of artifacts from eeg signals: A review," Sensors, vol. 19, no. 5, p. $987,2019$.

[17] W. K. So, S. W. Wong, J. N. Mak, and R. H. Chan, "An evaluation of mental workload with frontal eeg," PloS one, vol. 12, no. 4, p. e0174949, 2017.

[18] J. A. Urigüen and B. Garcia-Zapirain, "Eeg artifact removal-state-of-the-art and guidelines," Journal of Neural Engineering, vol. 12, no. 3, p. 031001, 2015.

[19] K. T. Sweeney, T. E. Ward, and S. F. McLoone, "Artifact removal in physiological signals—practices and possibilities," IEEE Transactions on Information Technology in Biomedicine, vol. 16, no. 3, pp. 488-500, 2012

[20] X. Chen, X. Xu, A. Liu, M. J. McKeown, and Z. J. Wang, "The use of multivariate emd and cca for denoising muscle artifacts from few-channel eeg recordings," IEEE Transactions on Instrumentation and Measurement, vol. 67, no. 2, pp. 359-370, 2017.

[21] N. E. Huang, Z. Shen, S. R. Long et al., "The empirical mode decomposition and the hilbert spectrum for nonlinear and non-stationary time series analysis," Journal Proceedings of the Royal Society of London. Series A: Mathematical, Physical Engineering Sciences, vol. 454, no. 1971, pp. 903-995, 1998.

[22] B. Mijović, M. De Vos, I. Gligorijević, J. Taelman, and S. Van Huffel, "Source separation from single-channel recordings by combining empirical-mode decomposition and independent component analysis," IEEE Transactions on Biomedical Engineering, vol. 57, no. 9, pp. 2188-2196, 2010.

[23] K. Zeng, D. Chen, G. Ouyang et al., "An eemd-ica approach to enhancing artifact rejection for noisy multivariate neural data," IEEE Transactions on Neural Systems Rehabilitation Engineering, vol. 24, no. 6, pp. 630-638, 2015.

[24] Z. Wu and N. E. Huang, "Ensemble empirical mode decomposition: A noise-assisted data analysis method," Journal Advances in Adaptive Data Analysis, vol. 1, no. 01, pp. 1-41, 2009.

[25] M. N. A. H. Sha'abani, N. Fuad, N. Jamal et al., "Development of cognitive and psychomotor task for eeg application with matlab-based gui," in IOP Conference Series: Materials Science and Engineering, 2020, vol. 917, no. 1, p. 012050: IOP Publishing.

[26] K. Kleifges, N. Bigdely-Shamlo, S. E. Kerick, and K. A. Robbins, "Blinker: Automated extraction of ocular indices from eeg enabling large-scale analysis," Frontiers in Neuroscience, vol. 11, p. 12, 2017.

[27] I. Zyma, S. Tukaev, I. Seleznov et al., "Electroencephalograms during mental arithmetic task performance," Data, vol. 4, no. 1, p. 14, 2019.

[28] J. R. Wolpaw and E. W. Wolpaw, Brain-computer interfaces: Principles and practice. OUP USA, 2012.

[29] R. Patel, M. P. Janawadkar, S. Sengottuvel, K. Gireesan, and T. S. Radhakrishnan, "Suppression of eye-blink associated artifact using single channel eeg data by combining cross-correlation with empirical mode decomposition," IEEE Sensors Journal, vol. 16, no. 18, pp. 6947-6954, 2016.

[30] M. H. Soomro, N. Badruddin, and M. Z. Yusoff, "Comparison of blind source separation methods for removal of eye blink artifacts from eeg," in 2014 5th International Conference on Intelligent and Advanced Systems (ICIAS), 2014, pp. 1-6: IEEE.

[31] J. Ferdous, S. Ali, E. Hamid, and K. I. Molla, "Sub-band selection approach to artifact suppression from electroencephalography signal using hybrid wavelet transform," International Journal of Advanced Robotic Systems, vol. 18, no. 1, 2021. 